colorful characters, and there can be children's books or a small aquarium in the waiting room [Figure 1].

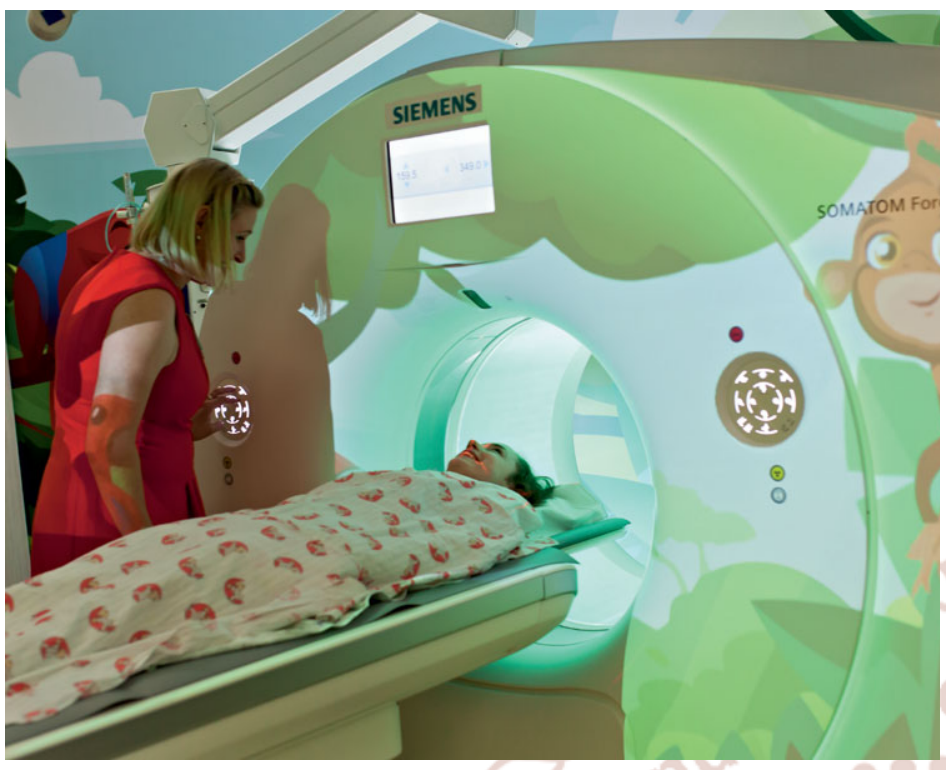

Figure 1: The investigation room and the gantry are colorful. This makes the environment friendly for the children and helps in receiving their cooperation

Insistence and support from parents is usually helpful. However, it may be necessary to sedate the child or use immobilizers [Figure 2] for longer studies such as MRI. Commonly used sedatives include diazepam, midazolam, and ketamine. Techniques and equipment should be employed to minimize the need for sedation as it has its own harmful effects. Where sedation or anesthesia is required, there should be dedicated pediatric specialists and recovery. The needs of parents should also be understood and addressed when considering any pediatric service.

\section{Equipment and protocols}

Dedicated pediatric imaging department with dedicated pediatric CT technologists may result in greater compliance with the pediatric protocols and significantly reduced patient dose. Facilities suitable for children ranging from premature infants to adult-sized teenagers are required, and these are often different from those used for adults.[4] Imaging needs to be child-focused and specific to the age of the child. Children must be considered in their own right, and not as small adults.[4]

There must be standardization of the techniques and various protocols used. Child-appropriate protocols should be documented and adhered to for all the modalities. Strict adherence to low-dose protocols can be challenging, particularly in a high-volume radiology department that scans both pediatric and adult patients.

\section{Training}

It must be ensured that only the staff with appropriate training is deployed and their performance is reviewed regularly. In reality, there is little incentive for radiographers to specialize in this area. As a result, few dedicated pediatric radiographers exist today. Also, radiologists reporting pediatric cases must have in-depth knowledge and expertise. Similar imaging findings in adult and pediatric cases may not be a disease in children or may represent some other disease, as pathologies afflicting children are different and peculiar than those in adults. Also, the anatomy is dynamic in children, making normal variants in children look like pathology. Proper knowledge of these variants helps avoid making these mistakes. The pediatric radiologist can offer the appropriate modality of choice. For example, neonate with vomiting and obstructive symptoms does not require a CT scan, as opposed to the adult population.

\section{Quality assurance}

Regular audits and quality checks for the equipment must be ensured for optimum performance and calibration for pediatric use.

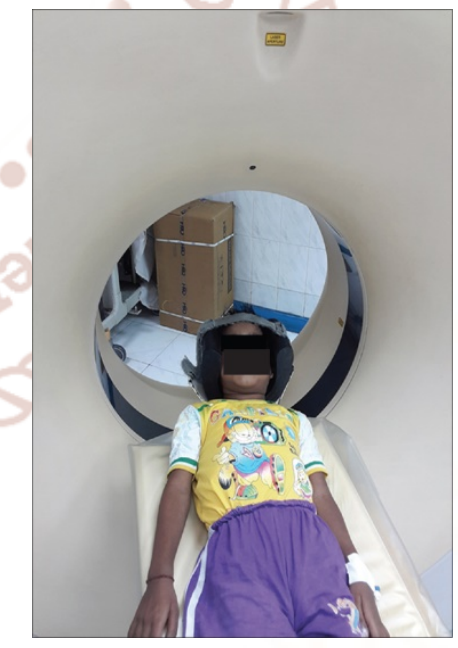

Figure 2: The head immobilizers should be used for children to avoid motion artifacts and repeat scan

\section{Radiation Protection}

Radiation protection and safeguarding are paramount concerns for this age group. Risk factor for cancer induction in children is about 10 times higher than in adults.[5-7] Also, children have longer life expectancy; therefore, they have a greater potential 
for manifestation of possible harmful effects of radiation [Figure 3]. On reviewing the literature,[8-10] there is suggestion that CT usage should be controlled and appropriate, and As Low As Reasonably Achievable (ALARA) principle should be strictly adhered to. Appropriate imaging modality should be used depending on the clinical indication (e.g. using USG (ultrasound) instead of CT in a suspected case of appendicitis). MRI is preferred over CT for most of the cross-sectional imaging workup in children, except for trauma evaluation.

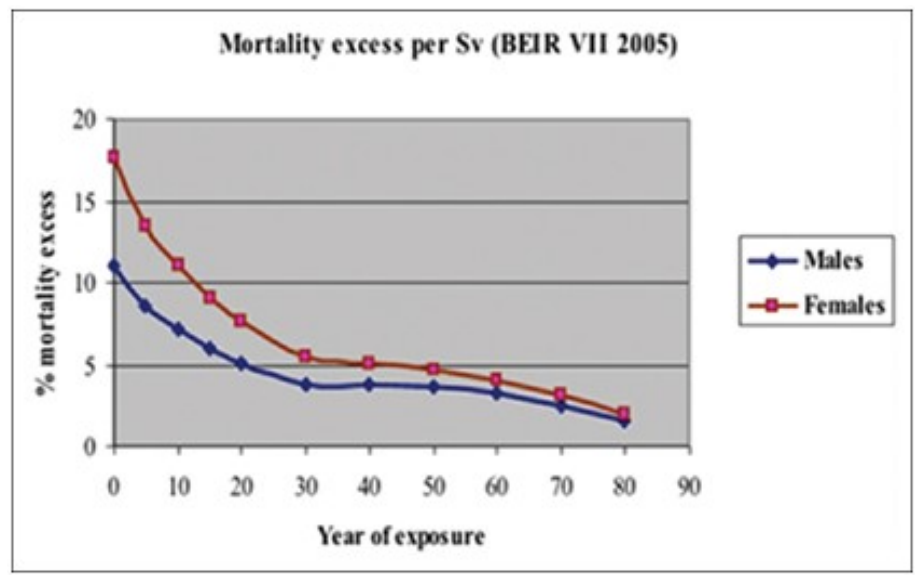

Figure 3: The graph depicts the percentage mortality excess due to radiation exposure with respect to the age at which the person is exposed. The younger the patient, higher is the percentage mortality excess. Also, it is higher for females as compared to males for the respective age groups

Various general issues which need to be taken care while using $\mathrm{X}$-ray equipment for imaging in children include the following:

- The generator used in the equipment should have enough power to allow short exposure times, should operate at a higher frequency, and should have large dynamic range of milliamperes and milliampereseconds levels

- Automatic exposure control (AEC) devices should be used with caution

- Beam filtration: The introduction of additional filtration in the $\mathrm{X}$-ray beam (commonly aluminum and copper filters) reduces the number of low-energy photons and, as a consequence, saves skin dose for the patients

- Anti-scatter grid: The anti-scatter grid in younger children gives limited improvement in image quality, as the irradiated volume is small, thus producing less of scattered radiation. Instead, it results in increased patient dose. Therefore, it should be removed. However, older children will still need the grid $\bullet$ Various protection devices like gonadal shield, thyroid shield, and breast shield must be used when appropriate.Proper collimation must be done. Only the area of interest must be exposed to radiation

- Proper factors must be used to avoid any repeat exposures

- To facilitate dose reduction in CT scan, the following need to be considered:

- Reducing tube voltage reduces the radiation dose

- An additional reduction in tube current further reduces the radiation dose

- The smaller an individual, the smaller are the anatomic features; so, higher-spatial-resolution CT scanning is required to visualize structures with the same precision as in the adult patient. Therefore, we should consider developing pediatric CT protocols to reduce the amount of radiation and obtain images of diagnostic quality. Various dose reduction protocols include ASIR from GE Healthcare, SAFIRE from Siemens, and iDose from Philips Healthcare

Low-dose imaging systems should be deployed using digital detection components, optimized for pediatric use

Multi-phasic studies should be avoided.

\section{Technical Specifics}

\section{Conventional Radiography}

There are existing policy guidelines regarding acceptable quality diagnostic radiography in the pediatric population which are set to ensure the triple objective of producing adequate and uniformly acceptable image, providing accurate radiological interpretation of the image, and using a reasonably low radiation dose per radiograph. To fulfill this triple objective, there are certain general recommendations which are as follows:

- Use of computed or digital radiography is recommended, so that exposure factors can be optimized and repeats are avoided

- Use of films of high contrast and capable of yielding high-resolution images

- Use of low absorption cassette or image plates and table-tops

- Exposure parameters should be stringent

- Adequate positioning, centering of beam, collimation, and restraining methods 
- Proper collimation devices with fine focus techniques to reduce the radiation dose without loss of detail

- Where possible, minimal projections must be used to visualize the area of interest

\section{Ultrasound}

Pediatric USG is relatively safe with no risks of radiation, cheap, and readily available. USG may be repeated over and over again for follow-up studies, without any significant risks. Special pediatric probes should be used which are usually smaller in size and have adjustable higher frequencies to cope with various depths and patientneeds.

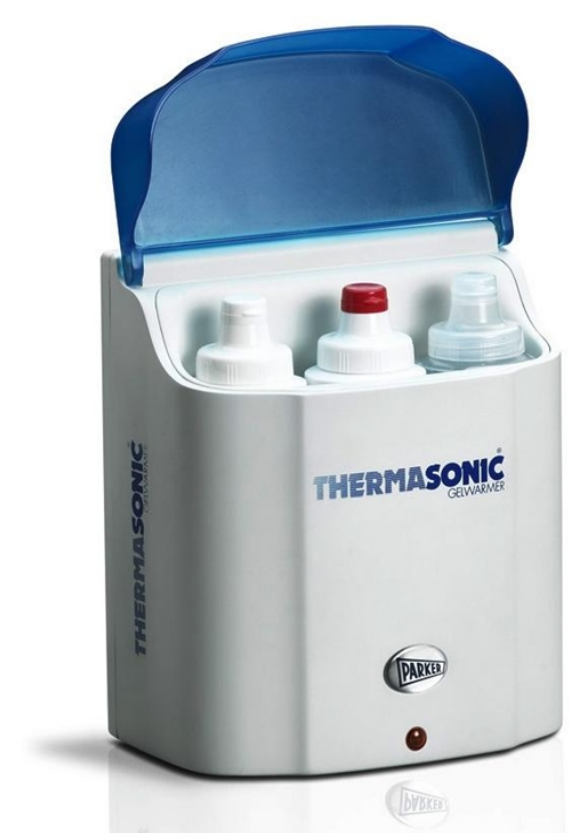

Figure 4: The gel warmers should be used in cold environment to avoid unpleasant sensation

The practice of warming the gel and towels is often desirable, which makes the process comfortable for the child [Figure 4]. Special care and aseptic procedures must be maintained to prevent the risk of infection to infants.

\section{Computed tomography}

The most important concern with the use of CT is about the radiation exposure. Various modifications in equipment design are recommended for optimization of scan parameters, which include:

○ Reduction in the rotation time (0.4- $0.5 \mathrm{~s}$ or less)

$\circ$ Reduced detector coverage commensurate to body size

○ Reduced field of view
○ Reduced kilovolts

○ Use of smart milliamperes/auto milliampere options.

The use of CT angiography also has several challenges in children. To perform successful angiography in children, factors like reduced contrast volume, injection rates, timing of scans, radiation dose minimization, and breath-holding abilities must be considered.

Adequate insulation of child from the room temperature (usually $18-22^{\circ} \mathrm{C}$ ) using blankets or warmers is also a must, especially in neonates who are vulnerable to develop hypothermia.

\section{Magnetic resonance imaging}

The major challenge in MRI is the need for sedation or general anesthesia in younger children.[11] Secondly, the relatively smaller anatomic structures in children create a challenge in terms of available signal and image resolution. So, higher signal-to-noise ratio is needed, which can be achieved using pediatric specific coils, high field strengths and by optimizing the field of view and slice thickness.

Various physiological factors also come into play while acquiring MR scan.[11] The rate of acquisition of images and contrast injection rates may be influenced by physiological changes in heart rate, breathing, and blood flow rates. All contrast studies should be power injected. Children may also find it difficult to hold their breaths and this may introduce artifacts during the image acquisition process. Acquisition of images using respiratory gating is helpful in such situations.[12]

Antenatal MRI is excellent for further evaluation of congenital anomalies detected on USG, but contrast agent is not administered to the pregnant mother. Antenatal MRI is best avoided in the first trimester.

Certain safety issues specific to children are also associated with MRI.[11] There is higher risk of radiofrequency heating effects due to poorly developed thermoregulatory mechanisms in children, high basal temperatures, and relatively higher surface area to weight ratio.[13] MR contrast agent should be avoided if possible, especially in children less than 2 years. 


\section{Clinical Applications}

\section{Pediatric brain}

USG is the preferred screening modality for infants as it is safe, cheap, easily available, and can be performed bedside. In some cases, cross-sectional imaging may be required for further evaluation. Also, in older children, USG is no longer useful once the frontals are closed and would require other cross $^{-}$sectional modalities like CT or MRI. MRI is the preferred cross-sectional modality in pediatric population. Structural and functional MR techniques are invaluable in investigating brain tissue anatomy and development.[14] CT is essentially reserved for trauma evaluation. For patients with ventriculo-peritoneal shunts, who require frequent imaging, the CT head is performed as per the "shunt protocol" which effectively is a very low-dose scan to provide the answers on issues like change in ventricle size and shunt catheter location. All other neurological indications are served by MRI (e.g. seizures, developmental delay, hypotonia, etc.).

\section{Pediatric chest}

Most common imaging technique employed for evaluation of chest is the radiograph. The challenges in acquiring radiographs include difficulty in achieving inspiration and likelihood of motional blur, wide range of tissue densities, and the need to minimize radiation dose.

Helical CT scans can produce remarkable information of the thoracic bony cage as well as vascular and pulmonary architecture simultaneously. To get the best images, one should allow short acquisition times, reduce motion artifacts, and allow dynamic contrast studies when needed.

MRI has the ability to clearly distinguish between mediastinal fat, blood vessels, and soft tissues. Cardiac anomalies, mediastinal masses, and chest wall lesions can be delineated with MRI. With the advent of newer MR imaging applications, functional lung imaging is now possible. One technique of functional MR imaging is fast MR imaging of the airway. Although experience is preliminary, dynamic airway abnormalities such as tracheobronchomalacia can be revealed noninvasively.[15]
Positron emission tomography/computed tomography (PET/CT) studies appear to be increasingly useful in lymphoma imaging in children.[16]

\section{Pediatric abdomen}

The plain abdominal radiograph allows initial assessment of a disease process. A specific methodology should be used to study the radiograph, so as make accurate diagnosis or to suggest the next useful imaging modality of choice. The neonatal gastrointestinal (GI) obstruction workup heavily relies on plain radiographs and fluoroscopic studies such an upper GI or contrast enema. Fluoroscopic studies should be performed with the pediatric settings, i.e. intermittent fluoroscopy mode instead of continuous, low frame rate, with appropriate collimation. Fluoroscopy is also used for intussusception reduction. USG is both safe and reliable to assess intra-abdominal organs.

Initial assessment of the liver, spleen, and kidneys is best performed with USG. It helps in determining the origin and extension of an abdominal mass. It is useful in differentiating medical from obstructive jaundice and in the initial suggestion of biliary atresia. In suspected cases of intestinal obstruction, USG helps in confirming the diagnosis and may also help in identifying the cause, e.g. intussusception. In cases of intussusception, it also helps in guiding hydro reduction. USG helps in diagnosing other GI conditions like appendicitis and genitourinary conditions like ovarian torsion and hemorrhagic cyst with high specificity.

$\mathrm{CT}$ has its use in the determination of injuries to organs following trauma or perforated viscus. The use of spiral CT techniques encourages better evaluation for acquired vascular abnormalities, vascular masses, and pre-operative evaluation of tumors.

The advances in MR techniques have significantly altered the investigation of abdominal and pelvic disease in children.[17] MRI helps in visualization of the biliary tract, pancreas, as well as intra- and extra-luminal bowel disease. MR urography is especially useful for anatomical and functional assessment of the urinary system.[18]

\section{Pediatric skeleton}

The imaging of skeletal structures typically starts with plain film radiography. Radiography plays an important role in detection and categorization of 
skeletal dysplasia. Diagnosis of bone tumors is also made on the plain radiograph. Additional modalities include MRI, which is usually used to stage the disease and for follow-up. The radionuclide bone scan using Tc-99m labelleddiphosphonates is one of the most commonly performed pediatric nuclear medicine procedures. Bone scintigraphy is used for diagnosis of bone and soft-tissue infection and can aid in the diagnosis of occult trauma without radiographic findings. There is a complimentary role for bone scintigraphy in the assessment of a child with suspected non-accidental injury. The use of bone scan in a child with unexplained bone pain or limp may provide a diagnosis that could be related to trauma, tumor, or inflammation. A negative bone scan can help relieve concern for significant pathology. Bone scans in children require careful attention to technique to obtain high-quality diagnostic images.

\section{Conclusion}

For proper utilization of imaging techniques in the pediatric population, it is important to address each step in the image-formation chain which includes: Image acquisition, image processing for display, and image review and assessment. The general guidelines for pediatric imaging departments would, therefore, include the following:

- Child should be at the center of all the decisions made

- There must be clinical justification for requesting any imaging investigation. The clinical benefits should outweigh any potential risk associated with the modality

- When possible, imaging evaluation of children should be performed in dedicated institutions

- The protocols for each modality must be specific and tailored to meet individual patient situations

- Radiation protection services must be available

- Optimization principles such as ALARA should be applied

- Specially trained radiographers, radiologists, and assisting staff should be available.

\section{Financial support and sponsorship}

Nil.

\section{Conflicts of interest}

There are no conflicts of interest.

\section{References}

1. Frush DP, Donnely LF, Rosen NS. Computed tomography and radiation risks: What pediatric health care providers should know. Pediatrics 2003;112:951-7.

2. Goske MJ, Applegate KE, Boylan J, Butler PF, Callahan MJ, Coley BD, et al. The 'Image Gently' campaign: Increasing $\mathrm{CT}$ radiation dose awareness through a national education and awareness program. PediatrRadiol 2008;38:265-9.

3. Erondu OF. Challenges and peculiarities of paediatric imaging.Medical Imaging in Clinical Practice. 2013.

4. NHS - National Imaging Board. Delivering quality Imaging services for Children. Available from: http://www.dh.gov.uk. [Last accessed on 2010 Mar 30].

5. Hall EJ. Lessons we have learned from our children: Cancer risks from diagnostic radiology. PediatrRadiol 2002;32:700-6.

6. BEIR (Committee on the Biological Effects of Ionising Radiation). Health Effects of Exposure of Low Levels of Ionizing Radiation. Washington DC: National Academy Press; 1990.

7. International Commission on Radiological Protection. 1990 recommendations of the International Commission on Radiological Protection. Oxford, England: Pergamon ICRP publication 60; 1991.

8. Brenner DJ, Elliston CD, Hall EJ, Berdon WE. Estimated risks of radiation-induced fatal cancer from pediatric CT. AJR Am J Roentgenol 2001;176:289-96.

9. Pearce MS, Salotti JA, Little MP, McHugh K, Lee $\mathrm{C}$, Kim KP, et al. Radiation exposure from CT scans in childhood and subsequent risk of leukaemia and brain tumours: A retrospective cohort study. Lancet 2012;380:499-505.

10. Mathews JD, Forsythe AV, Brady Z, Butler MW, Goergen SK, Byrnes GB, et al. Cancer risk in 680000 people exposed to computed tomography scans in childhood or adolescence: Data linkage study of 11 million Australians. BMJ 2013;346:f2360. 
11. Ditchfield M. 3T MRI in paediatrics: Challenges and clinical applications. Eur J Radio 2008;68:309-19.

12. Mitchell CL, Vasanawala SS. An approach to pediatric liver MRI. AJR Am J Roentgenol 2011;196:W519-26.

13. Machata AM, Willschke H, Kabon B, Prayer D, Marhofer P. Effect of brain MRI on body core temperature in sedated infants and children. $\mathrm{Br} \mathrm{J}$ Anaesth 2009;102:385-9.

14. Raschle N, Zuk J, Ortiz-Mantilla S, Sliva DD, Franceschi A, Grant PE, et al. Pediatric neuroimaging in early childhood and infancy: Challenges and practical guidelines. Ann $\mathrm{N}-\mathrm{Y}$ AcadSci 2012;1252:43-50.
15. Frush DP, Donnelly LF, Chotas HG. Contemporary pediatric thoracic imaging. AJR Am J Roentgenol 2000;175:841-51.

16. Riad R, Omar W, Kotb M, Hafez M, Sidhom I, Zamzam M, et al. Role of PET/CT in malignant pediatric lymphoma. Eur J Nucl Med Mol Imaging 2010;37:319-29.

17. Darge K, Anupindi SA, Jaramillo D. MR imaging of the abdomen and pelvis in infants, children and adolescents. Radiology 2011;261:12-29.

18. Michael R. Potential of MR-imaging in the paediatric abdomen. Eur J Radiol 2008;68:235-44.

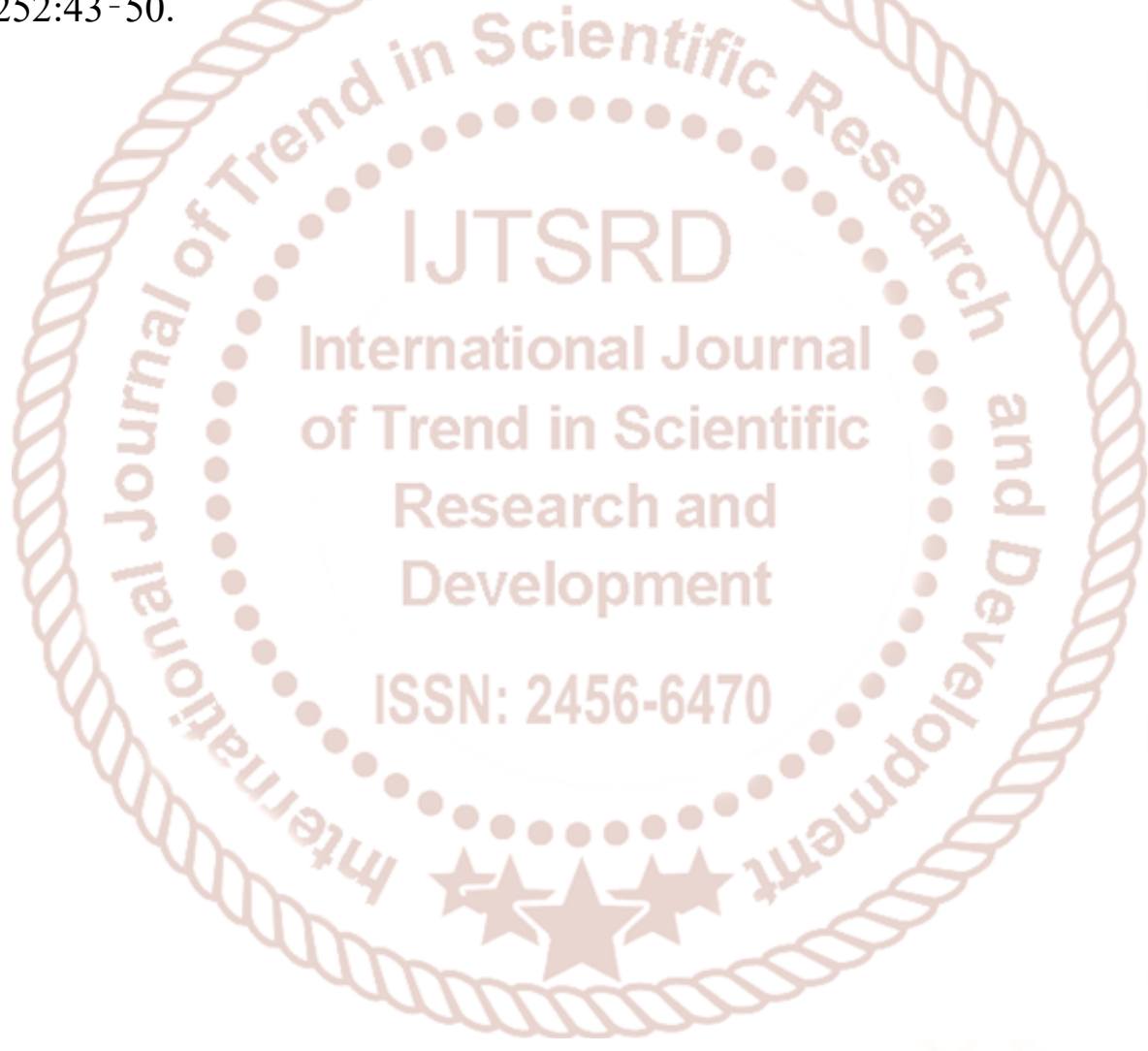

